\title{
$k$ 连通无爪图中坆长的周
}

本文所涉及的图都是有限无向简单图. 设 $G$ 是一个图, 总用 $V(G) 、 E(G)$ 分别表示 $G$ 的顶点集、边集，而 $p-|V(G)|$. 设 $U \subseteq$ $V(G)$, 总用 $G[U]$ 表示 $G$ 中由 $U$ 导出的子 图. 图 $G$ 称为无爪的, 如果对于任意 $U \subseteq$ $V(G)$, 总有 $G[U] \neq K_{1,3}$ 。图 $G$ 称为 $m$ 路 连通, 如果对于任意 $\{u, v\} \subseteq V(G)$, 总有长 至少为 $m$ 的 $(u, v)$ 路.

1960 年 O. Ore 证明了：若图 G中任意 两个不相邻的顶点 $v_{1} 、 v_{2}$, 有

$$
d\left(v_{1}\right)+d\left(v_{2}\right) \geqslant p,
$$

则 $G$ 是 Hamilton 图. 1980 年 J. A. Bondy 推广了上述结论,给出了: 设 $G$ 是 $k$ 连通图, $C$ 是 $G$ 中最长的圈, $R=V(G) \backslash V C)$. 若 对于 $G$ 中任意 $k+1$ 个顶点的独立集 $\left\{v_{1}\right.$, $\left.v_{2}, \cdots, v_{k+1}\right\}$, 有 $\sum_{i=1}^{k+1} d\left(v_{i}\right) \geqslant p+k(k-1)$, 则 $G[R]$ 不含 $(k-1)$ 路连通的子图. 从 而,也不含 $K_{k}$ 。

1986 年田丰等讨论了无爪图为 HamiIton 图的充分条件, 证明了: 设 $G$ 是 2 连通 的无爪图. 若对于 $G$ 中任意 3 个顶点的独立 集 $\left\{v_{1}, v_{2}, v_{3}\right\}$, 有 $d\left(v_{1}\right)+d\left(v_{2}\right)+d\left(v_{3}\right) \geqslant$ $p-2$, 则 $G$ 是 Hamilton 图. 本文将对此结
论作类似于上述的推广,证明了下列

定理设 $G$ 是 $k$ 连通的无爪图, $k \geqslant 2$ ， $C$ 是 $G$ 中最长的圈， $R=V(G) \backslash V(C)$. 若 对于 $G$ 中任意 $k+1$ 个顶点的独立集 $\left\{v_{1}\right.$, $\left.v_{2}, \cdots, v_{k+1}\right\}$, 有

$$
\sum_{i=1}^{k+1} d\left(v_{i}\right) \geqslant p+k(k-3),
$$

则 $G[R]$ 不含 $(k-2)$ 路连通的子图. 从 而,也不含 $K_{k-1}$.

推论 设 $G$ 是 $k$ 连通的无爪图, $k \geqslant 2$ ， $C$ 是 $G$ 中最长的圈， $R=V(G) \backslash V(C)$. 若 $G$ 中的最小次

$$
\delta \geqslant \frac{1}{k+1}(p+k(k-3)),
$$

则 $G[R]$ 不含 $(k-2)$ 路连通的子图. 从 而,也不含 $K_{k-1}$.

上述推论推广了 M. M. Matthews 等人 在 1985 年所得的下列结论: 设 $G$ 是 2 连通 的无爪图. 若 $G$ 的最小次 $\delta \geqslant \frac{1}{3}(P-2)$, 则 $G$ 是 Hamilton 图.

吴正声

(南京师范大学数学系)

\section{双下标相依变是和的大数律}

本文讨论了双下标相依随机变量和，对 广义高斯序列,得到了重对数律的形式.

设 $\left\{a_{n j}: n \geqslant 1,-\infty<i<\infty\right\}, A_{n}=$ $\sum_{i=-\infty}^{\infty} a_{n i}<\infty, \lim _{n \rightarrow \infty} A_{n}=\infty,\left\{\varepsilon_{i}:-\infty<i<\right.$ $\infty\}$ 为随机变量列, $S_{i}=\sum_{i=-\infty}^{\infty} a_{n i} \varepsilon_{i}$.
定理 1 设 $\left\{\varepsilon_{i}, \mathbb{F}_{i} ;-\infty<i<\infty\right\}$ 为局部广义高斯序列, $\alpha\left(\varepsilon_{i} \leqslant K<\infty\right.$, $(-\infty<i<\infty)$, (i) 如存在常数 $c_{i} \geqslant 0$, $d>0$, 使

$$
\begin{aligned}
& A_{m, n}=\sum_{i=-\infty}^{\infty}\left(a_{m i}-a_{m i}\right)^{2} \leqslant\left(\sum_{k=m+1}^{n} c_{k}\right)^{d}, \\
&\left(n>m \geqslant m_{0}\right) ;
\end{aligned}
$$




$$
\left(\sum_{i=m_{0}}^{n} c_{i}\right)^{d}-O\left(A_{n}\right)
$$

找 (ii) $A_{m, n} \leqslant h(n-m),\left(n>m \geqslant m_{0}\right)$; $h(n)=O\left(A_{n}\right)$, 存在 $\lambda>0$ 及某整数 $k \geqslant 2$, 使 $\varliminf_{n \rightarrow \infty} h(k n) / h(n) \geqslant k^{2}$, 对任 $r>0$, 存在 $\rho<1$, 使 $\varlimsup_{n \rightarrow \infty}\left\{\max _{\rho n<i<n} h(i) / h(n)\right\}<1+r$, 这里 $h(x) \geqslant 0$, 则

$$
\varlimsup_{n \rightarrow \infty}\left|S_{n}\right| /\left(2 A_{n} \log \log A_{n}\right)^{1 / 2} \leqslant K \text {, a.s. }
$$

系 1 (i): 如 $\left\{\boldsymbol{s}_{i}:-\infty<i<\infty\right\}$ 为 乘法序列, $\left|8_{i}\right| \leqslant 1$, a. s. $(-\infty<i<\infty)$, 则在定理其它条件下其结论对 $K=1$ 成立; (ii) 如 $\left\{\varepsilon_{i}:-\infty<i<\infty\right\}$ 为独立高斯序 列, $\varepsilon_{k} \sim N\left(0, \sigma_{k}^{2}\right)$, $\sup _{k} \sigma_{k}^{2}<\infty$, 则在定理 其它条件下其结论对 $K=\sup _{k} \sigma_{k}^{2}$ 成立；(iii) 如 $\left\{\varepsilon_{i}, \mathscr{F}_{i}, i \geqslant 1\right\}$ 为局部广义高斯序列, $\alpha\left(\varepsilon_{i}\right) \leqslant K<\infty,(i \geqslant 1), D_{n}=\sum_{k=1}^{n} a_{k}^{2} \rightarrow$ $\infty$, 则

$$
\begin{array}{r}
\varlimsup_{n \rightarrow \infty}\left|\sum_{k=1}^{n} a_{k} \varepsilon_{k}\right| /\left(2 D_{n} \log \log D_{n}\right)^{1 / 2} \\
\leqslant K \text {. a. s.. }
\end{array}
$$

(iv) 如 $\left\{\varepsilon_{i}, \mathscr{F}_{i}, i \geqslant 1\right\}$ 为局部广义高斯 序列, $\alpha\left(\varepsilon_{i}\right) \leqslant K<\infty$, 设 $f \in L^{2}(0,1)$, 使

$$
\begin{aligned}
& \lim _{n \rightarrow \infty} n^{-1} \sum_{i=1}^{n} f^{2}(i / n)=\int_{0}^{1} f^{2}(t) d t>0, \\
& \varlimsup_{i \rightarrow 1}|f(t)|<\infty,
\end{aligned}
$$

存在 $L>0, m_{0} \geqslant 1,0<\theta_{0}<1$ 使

$$
\begin{gathered}
m^{-1} \sum_{i=1}^{m}(f(i / m)-f(i / n))^{2} \leqslant L\left(1-\frac{m}{n}\right), \\
\left(n>m_{0}, \theta_{0} \leqslant m / n<1\right),
\end{gathered}
$$

则

$$
\varlimsup_{n \rightarrow \infty}\left|\sum_{i=1}^{n} f(i / n) \varepsilon_{i}\right| /(2 n \log \log n)^{1 / n}
$$

$$
\leqslant K\left(\int_{0}^{1} f^{2}(t) d t\right)^{1 / 2} \text { a.s. }
$$

定理 2 设 $\left\{\varepsilon_{i}:-\infty<i<\infty\right\}$ 满足 $\left|E\left(\varepsilon_{i} \varepsilon_{j}\right)\right| \leqslant \rho(j-i)\left(E\left|\varepsilon_{i}\right|^{s}\right)^{1 / s}$

$$
\text { - }\left(E\left|\varepsilon_{i}\right|^{r}\right)^{1 / r},(j>i) \text {, }
$$

此处 $r \geqslant 2, \frac{1}{r}+\frac{1}{s}=1, \sum_{i=1}^{\infty} \rho(i)<\infty$, $\sum_{\substack{i \neq-\infty \\ i \neq 0}}^{\infty}\left(\log ^{2}|i|\right) a_{n i}^{2}\left(E\left|\varepsilon_{i}\right|^{r}\right)^{2 / r}<\infty, \sup _{i} E\left|\varepsilon_{i}\right|^{r}<$ $\infty$, (i) 存在 $c_{i} \geqslant 0, d$, 使 $d r>2, A_{n, n} \leqslant$ $\left(\sum_{k=m+1}^{n} c_{k}\right)^{d},\left(n>m \geqslant m_{0}\right),\left(\sum_{i=m_{0}}^{n} c_{i}\right)^{d}=$ $O\left(A_{n}\right)$, 则对任 $\delta>2 /(d r-2), \lim _{n \rightarrow \infty}\left|S_{n}\right| \mid$ $A_{n}^{1 / 2}\left(\log A_{n}\right)^{(1+\delta) / r}=0$, a.s. 对任 $a>0$, $a r / 2>1, \lim _{n \rightarrow \infty}\left|S_{n}\right| / A_{n}^{1 / 2}\left(\log A_{n}\right)^{a / 2}=0$, a.s. (ii) 对 $\lambda>1,\left\{a_{n i}\right\}$ 满足定理 1 的条件(ii), 则定理 2 (i) 的结论对 $\delta>1 /(\lambda-1)$ 成立.

秉 2 设 $\left\{\varepsilon_{i}:-\infty<i<\infty\right\}$ 为平稳 Q混合序列, 存在 $r \geqslant 2, \frac{1}{r}+\frac{1}{s}=1$, 使 $\sum_{i=1}^{\infty} Q^{1 / r}(i)<\infty, E\left(\varepsilon_{i}\right)=0,(-\infty<i<$ $\infty), \sup _{i} E\left|e_{i}\right|^{r}<\infty, \sum_{i=-\infty}^{\infty}\left(\log ^{2}|i|\right) a_{m i}^{2}$. $\left(E\left|\varepsilon_{i}\right|^{r}\right)^{2 / r}<\infty$, 则在定理 2 (i) 或 (ii) 条件 下,其结论成立。

胡合朱力行 （安数大学数学系,合肥） 苏 注 (中国科学技术大学数学系,合肥)

\section{毎个自对偶的偏序集都有自对偶的全序扩充}

众所周知，每一个偏序集都有一个全序 扩充 ${ }^{[1]}$, 由此导致了众多的作者确定偏序集
的什么性质可以在某一(或任一)全序引゙充中 得以保持 (例如见文献 [2]). 本文的结果对 